\title{
Leishmaniasis cutánea en la provincia del Magdalena Centro, Cundinamarca, Colombia. Una serie de casos
}

\section{Cutaneous Leishmaniasis in the region of Magdalena Centro in the Department of Cundinamarca, Colombia. A Series of Cases}

\author{
Hernando Gaitán LeE \\ Hospital San Vicente de Paul de San Juan de Rioseco, \\ Colombia \\ Giovana Lasso Araujo \\ Pontificia Universidad Javeriana, Colombia \\ Hernando Gaitán Duarte \\ Universidad Nacional de Colombia, Colombia
}

Cómo citar: Gaitán Lee H, Lasso Araujo G, Gaitán Duarte H. Leishmaniasis cutánea en la provincia del Magdalena Centro, Cundinamarca, Colombia. Una serie de casos. Univ Med. 2017;58(2):1-4. doi: https://doi.org/10.11144/Javeriana.umed58-2.leis

\section{RESUMEN}

El objetivo del artículo es describir las diferentes características demográficas y clínicas de una serie de pacientes con leishmaniasis cutánea en un municipio de la región del Magdalena Centro del departamento de Cundinamarca Colombia. Bituima es un municipio ubicado entre 1250 y $2250 \mathrm{msnm}$, el cual en su mayoría tiene población rural. Se reportan trece casos de pacientes con leishmaniasis cutánea, diagnosticados entre agosto del 2015 y mayo del 2016, en el puesto de salud de Bituima, institución que atiende pacientes con aseguramiento subsidiado por el Estado. La presentación clínica inicial fue variada; sin embargo, todos los casos se presentaron a la primera consulta médica con úlcera indolora. Se encuentra un periodo de casi dos meses entre la aparición de la lesión y la primera consulta médica. Los casos se confirmaron mediante frotis directo de Leishmania y se dio tratamiento con Glucantime. En conclusión, se informa una alta frecuencia de la enfermedad en este periodo en el municipio. Se deben fortalecer las estrategias para acortar el tiempo entre la aparición de la úlcera y la primera valoración médica.

Palabras clave

leishmaniasis; cutánea; Colombia; lutzomyia.

\section{ABSTRACT}

The objective of this research is to describe the different demographic and clinical characteristics of a series of patients with Cutaneous Leishmaniasis in a municipality in the Magdalena Center region of the department of Cundinamarca, Colombia. In the present article we reported 13 patients with cutaneous Leishmaniasis in a period of time between August 2015 and May 2016, in a primary care center located in Bituima where people with insurance subsidized by the state are attended. This municipality is located between 1250 and 2250 meters over the sea level, 2 hours far from Bogota, the capital of Colombia. The population is mainly rural located out of the town. The initial clinical presentation was varied, however, all the cases presented at the first medical visit 
with painless ulcer. There is a period of almost two months between the appearance of the injury and the first medical appointment. Cases were confirmed by direct leishmaniasis, treatment with Glucantime was given. In conclusion, a high frequency of the disease is reported in this period of time in the municipality. Strategies should be strengthened to shorten the time between the onset of the ulcer and the first medical evaluation.

Keywords

leishmaniasis; cutaneous; Colombia; lutzomyia.

\section{Introducción}

La leishmaniasis es una zoonosis ocasionada por un protozoario flagelado del género Leishmania, el cual ingresa al organismo a través de la picadura de insectos flebótomos de la especie Lutzomyia. El protozoario parasita los macrófagos, afecta la piel, las mucosas o las vísceras, dependiendo de la cepa infectante y de la respuesta inmune del hospedero [1]. Se ha demostrado la presencia de seis especies de Leishmania en Colombia y se han descrito 125 especies de flebotomíneos, aunque solo siete de ellas se han encontrado naturalmente infectadas con Leishmania e incriminadas como vectores. Los focos endémicos se distribuyen a lo largo y ancho del territorio nacional, centrados en las regiones rurales que incluyen zonas selváticas (Amazonía y costa del Pacífico), áreas de bosque seco tropical (costa atlántica), cultivos de café (región andina), desiertos y llanos (península de La Guajira y Llanos Orientales) [2].

La notificación en la década de los noventa era de aproximadamente 6500 casos nuevos, con un aumento progresivo hasta alcanzar alrededor de los 20.000 casos anuales en 2005 y 2006 [3]. En 2015 se reportaron 7900 casos confirmados de leishmaniasis, distribuidos así: 7777 casos $(98,4$ \%) de leishmaniasis cutánea, 108 casos $(1,4$ $\%)$ de leishmaniasis mucosa y 15 casos $(0,2$ $\%)$ de leishmaniasis visceral [4], por lo que es considerado un problema de salud pública para el país.

Las manifestaciones clínicas de la leishmaniasis cutánea son variadas: desde pápulas, nódulos, placas de aspecto verrugoso, hasta lesiones ulceradas. La presentación más frecuente en Colombia es la úlcera indolora con afectación linfangítica y adenopatía regional [5].

La guía del Ministerio de Salud y Protección Social en Colombia establece que el diagnóstico de leishmaniasis cutánea debe realizarse mediante tres frotis directos de la lesión (sensibilidad del $85 \%$ al $90 \%$ en pacientes que no superen los cuatro meses de evolución). En caso de presentar cualquier muestra positiva, se debe iniciar el tratamiento. Por otro lado, en caso de presentar las tres muestras negativas, es precisa una biopsia de la lesión (la detección del ADN parasitario tiene una sensibilidad mayor al $70 \%)[6]$.

El tratamiento de primera línea consta de sales de antimonio pentavalente, las cuales se administran de manera intramuscular durante 20 días a una dosis de $20 \mathrm{mg} / \mathrm{kg} /$ día. Previo al inicio del tratamiento, es necesario tomar pruebas de función hepática renal, pancreática y electrocardiograma, debido a la potencial toxicidad del medicamento sobre hígado, páncreas, riñón y corazón [6].

La provincia del Magdalena Centro es una zona endémica para esta patología. En el presente artículo reportamos una serie de 13 casos de leishmaniasis cutánea diagnosticados en un periodo de 8 meses, con dos objetivos: 1) hacer una aproximación a las características demográficas y clínicas de los pacientes con esta infección en un área cercana a Bogotá y 2) describir aspectos relacionados con los antecedentes de los pacientes, el cuadro clínico, el tratamiento empleado y el seguimiento.

\section{Descripción de los casos}

Lugar: los casos se presentaron en Bituima, municipio ubicado en el departamento de Cundinamarca, en la provincia del Magdalena Centro (hacen parte de esta región los municipios de San Juan de Rioseco, Bituima, Guayabal de Síquima, Vianí, Chaguaní, Pulí y Beltrán). Está localizado $94 \mathrm{~km}$ al occidente de Bogotá. La cabecera municipal se encuentra localizada a $1412 \mathrm{msnm}$ y tiene una temperatura media de 23 
oC. El área rural abarca desde cerca de 1250 hasta $2250 \mathrm{msnm}$ [7].

Sitio de atención: todos los pacientes fueron atendidos en el puesto de salud de Bituima, centro de atención primaria, parte de la red de instituciones dependientes del Hospital San Vicente de Paúl de San Juan de Rioseco. Se realizó un muestreo consecutivo estricto de los pacientes atendidos entre agosto de 2015 y mayo de 2016.

Variables: edad, peso, ocupación, sitio de residencia, localización de la lesión, estado de la lesión al finalizar el tratamiento, tiempo entre la aparición de la lesión y la consulta médica inicial, tiempo de curación, comorbilidades, adherencia al manejo.

Consideraciones éticas: se garantizó la confidencialidad de la información y la privacidad de los pacientes. Se solicitó la autorización y se firmó un consentimiento informado para la publicación de su información y sus fotografías en la serie de casos. No hay Comité de Ética Institucional en el Hospital San Vicente de Paúl de San Juan de Rioseco.

\section{Discusión y resultados}

Pese a que la leishmaniasis es una entidad endémica de notificación obligatoria en Colombia, la literatura médica publicada en revistas médicas locales es escasa. Entre los estudios descriptivos se encuentra el realizado por Vélez y colaboradores [5], en Antioquia, el cual informa 27 casos, pero en un periodo de 18 años.

En cuanto a la georreferenciación de los casos, podemos encontrar que estos se concentran en su mayoría en la región sur del municipio (10 de los 13 casos). De esta región, las dos veredas más afectadas son Gualiva y Montaña, con tres casos cada una, seguidas de Palo Blanco Bajo y Pajitas con dos casos y Palo Blanco Alto y Progreso con un caso cada una (figura 1). La información detallada de las características de los pacientes se presenta en la tabla 1 .
Tabla 1. Características de los pacientes atendidos con leishmaniasis cutánea

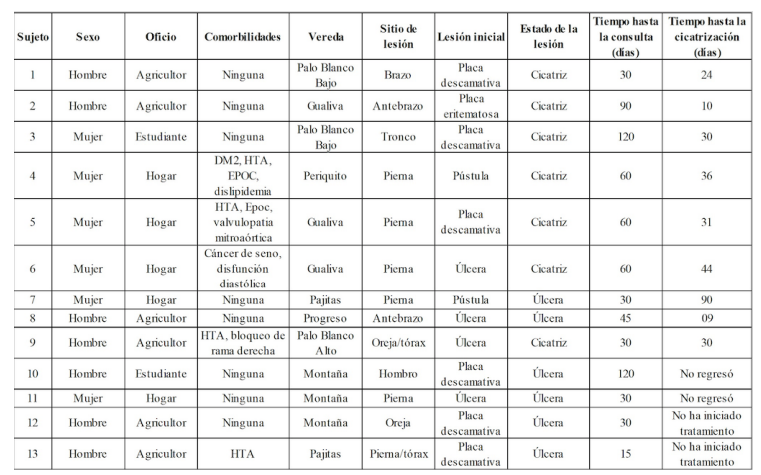

Nota:DM2: diabetes mellitus 2; HTA: hipertensión arterial; EPOC: enfermedad pulmonar obstructiva crónica.

La mediana del tiempo que transcurre entre la aparición de la sintomatología y la primera valoración médica es de 52,5 días, con un mínimo de 30 días y un máximo de 120. Seis de los pacientes reportaron como lesión inicial una placa descamativa; cuatro, una lesión ulcerada; dos, una pústula, y uno, una placa eritematosa. El $100 \%$ de los pacientes se presentaron a la primera consulta médica con una lesión ulcerada (figura 2). Esta demora sugiere la necesidad de fortalecer y realizar campañas educativas para favorecer un rápido conocimiento de los síntomas y asistencia oportuna a la consulta médica [3]

Nueve de once recibieron tratamiento con Glucantime al momento de escribir este informe. Todos presentaron curación clínica. El tiempo de curación oscila entre 9 y 90 días con una mediana de 30 días. En dos pacientes no se había iniciado tratamiento. Uno, debido a que el primer frotis dio negativo, debió ser llevado a un segundo frotis, el cual resultó positivo [3,6]. El otro paciente no había iniciado tratamiento, debido a que cuenta con enzimas hepáticas elevadas y tiene pendiente valoración por parte del servicio de medicina interna.

Además, se encontró una baja frecuencia de efectos adversos asociados al tratamiento con Glucantime. En general, es clínicamente bien tolerado, sin evidencia de efectos secundarios importantes en ninguno de los pacientes sometidos a este. Entre los pacientes tratados, uno presentó un cuadro clínico sugestivo de síndrome coronario agudo, el cual fue descartado 
después de la realización de los estudios diagnósticos pertinentes.

Es importante mencionar el difícil seguimiento de los pacientes, quienes posterior a la finalización del tratamiento, en su mayoría, no regresan a consulta médica. Fue necesaria la visita domiciliaria a todos. Además, es importante mencionar que únicamente cinco de los once se realizaron los exámenes paraclínicos de control, pese a que se solicitaron en múltiples oportunidades, ya que las características geográficas del municipio y la alta tasa de dispersión limitan el acceso a los servicios de salud de los habitantes.

\section{Conclusiones}

La leishmaniasis es una de las múltiples enfermedades endémicas transmitidas por vectores en la región del Magdalena Centro y departamento de Cundinamarca, que representa en esta región un problema de salud pública, en especial para el municipio de Bituima, dada el alto número de casos que se presentaron durante un año. Se deben implementar estrategias para que los pacientes consulten tempranamente ante la clínica sugestiva de esta enfermedad.

\section{Referencias}

1. Organización Panamericana de la Salud. Leishmaniasis cutánea y mucosa [internet]; 2017. Disponible en: http://www.who.int/m ediacentre/factsheets/fs375/es/

2. Vélez ID, Hendrickx E, Robledo SM, Agudelo $S$ del P. Leishmaniasis cutánea en Colombia y género. Cad Saúde Pública (Rio de Janeiro). 2001;17(1):171-80.

3. Zambrano P. Protocolo de vigilancia en salud pública: leishmaniasis [internet]. Bogotá: Instituto Nacional de Salud; 2014. Disponible en: http://www.ipsunipamplona.com/es/im ages/sampledata/sivigila_2015/protocolos_ epidemiologicos/PRO\%20Leishmaniasis.p df
4. Instituto Nacional de Salud. Boletín Epidemiológico Semanal. 2015;(52).

5. Vélez I, Jiménez A, Vásquez D, Robledo SM. disseminated cutaneous leishmaniasis in Colombia: Report of 27 Cases. Case Rep Dermatol. 2015 Oct 7;7(3):275-86.

6. Echeverry M, Gaona J, Gualtero S, Agudelo $S$, et al. Guía de atención de la leishmaniasis: guías de práctica clínica [internet]. Bogotá: Misterio de Salud y Protección Social; 2007. Disponible en: http://www.acin.org/acin/new/Portals/0 /Templates/Guia\%20Leishmania.pdf

7. Alcaldía de Bituima-Cundinamarca. Bituima atractiva: nuestro municipio [internet]. Disponible en: http://www.bituimacundina marca.gov.co/informacion_general.shtml 